\title{
From spinons to magnons in explicit and spontaneously dimerized antiferromagnetic chains
}

\author{
Ariel Dobry \\ Departamento de Física, Universidad Nacional de Rosario, \\ and Instituto de Física Rosario, Avenida Pellegrini 250, \\ 2000 Rosario, Argentina \\ David Ibaceta \\ Instituto de Astronomía y Física del Espacio, \\ Casilla de Correos 67, Sucursal 28, \\ 1428 Buenos Aires, Argentina.
}

We reconsider the excitation spectra of a dimerized and frustrated antiferromagnetic Heisenberg chain. This model is taken as the simpler example of compiting spontaneous and explicit dimerization relevant for Spin-Peierls compounds. The bosonized theory is a two frequency Sine-Gordon field theory. We analize the excitation spectrum by semiclassical methods. The elementary triplet excitation corresponds to an extended magnon whose radius diverge for vanishing dimerization. The internal oscilations of the magnon give rise to a series of excited state until another magnon is emited and a two magnon continuum is reached. We discuss, for weak dimerization, in which way the magnon forms as a result of a spinon-spinon interaction potential.

One dimensional antiferromagnetic systems have attracted a great deal of interest over the last decades. They are typical examples where the low dimensionality enhances quantum fluctuations producing effects completely different than the ones expected by classical theories of magnetism. The interest in one-dimensional magnetic system have recently been strongly renewed in view of the discovery of a new class, in general inorganic compounds, with definite one-dimensional character . Detailed spectral characterizations are now avaible and the theory is pushed to make precise predictions on how different microscopic interactions influence the spectral response of this system. We discuss in this work the case of a gapped antiferomagnetic chain and analyze how an explicit dimerization and a frustrated interaction compete between them and how the magnetic spectra evolve with the microscopic parameters. A similar subject has recently been analized by different numerical technics 1 , 2 . An analytical approach for the case of weak dimerization limit has been also proposed 3 . We will discuss the similarities and differences with our results. We take the recently discovered inorganic material $\mathrm{CuGeO}_{3} \mathrm{O}$ as a reference even though our result could be more general. Our started model Hamiltonian reads:

$$
\frac{H}{J}=\sum_{i}\left\{\left(1+\delta(-1)^{i}\right) \mathbf{S}_{i} \cdot \mathbf{S}_{i+1}+\alpha \mathbf{S}_{i} \cdot \mathbf{S}_{i+2}\right\}
$$

where $J$ is the nearest-neighbor (NN) exchange coupling, $\alpha$ the frustration parameter and $\delta$ measures the amount of the explicit dimerization. There are two different motivations to associate this model-Hamiltonian with the magnetic spectra of a Spin-Peierls compound. First, the temperature dependence of the magnetic susceptibility of $\mathrm{CuGeO}_{3}$ could only be accounted for if an important next nearest-neighbor (NNN) is included in the 1D Heisenberg model used to describe this material. 0 lo It is therefore natural to include an explicit dimerization on the exchange interaction to describe the magnetic excitations in the low temperature Peierls phase and this has been in fact the approach used in various theoretical studies $\mathbf{0}$, Second, if the adiabatic hypothesis is left out, the system turn to be a coupled spin-phonon one. Even though the magnetic interactions have mainly one-dimensional character they interact with the three dimensional phonons. The excitations of the low temperature phase are strongly affected by the interchain elastic coupling. In fact, topological solitons (kink) which are genuine excitations of the isolated chain cannot longer exist as free excitations because they create a zone with opposite dimerization phase relative to the surrounding chains. A kink and an antikink are strongly interacting excitations. Their interactions were affounted in previous work by a linear confined potential 3 - or as producing a kind of domain configuration where the kink and antikink oscillate around an equilibrium distance 10 . As it has been recently remarked the model Hamiltonian (11) is the simplest example where this processes could be studied. In this context the first term of Eq. (17) account for the explicit dimerization imposed by neighboring chains and the second term for the tendency to spontaneous dimerization as we will discuss in the following.

For $\alpha=0$ and strong dimerization $(\delta)$ the ground state is a product of singlet over the strong bonds. The first excitations correspond to promote a singlet to a triplet and then delocalize it to build states of definite momentum. They form a band of spin-1 magnon excitations. In the opposite limit $(\delta=0)$ translational symmetry is not explicitly broken. However for $\alpha$ greater than a critical value $\left(\alpha_{c} \sim 0.23\right)$ the system spontaneously dimerize and a gap in the spectra is open. At 
$\alpha=1 / 2$ (Majumdar-Ghosh point) the exact ground state is the double degenerate product of singlets over the nn bonds. The excitations have been variationally evaluated by Shastry and Sutherland11 (SS). They are massive $S=1 / 2$ spinons which correspond to an uncoupled spin separating two regions of singlet dimers. The two previously discussed cases represent the extremal situation of correlation length equal to the lattice constant. As we discuss below the structure of the lower energy triplet excitations remains similar for other values of the correlation length in the limits previoulsy discussed.

The main question we address in this work is how the spectra evolve from one limit to another i.e. how the massive spinons bound in a magnon. To address this question we analyze this system in the limit of small $\delta$ and $\alpha$ slightly larger than the critical value. In this region the low energy spectrum could bestudied by bosonization. The bosonized Hamiltonian is 14 :

$$
\begin{array}{r}
H_{b o s}=\int d x\left\{\frac{u \beta^{2}}{2} \Pi^{2}+\frac{u}{2 \beta^{2}}\left(\partial_{x} \phi\right)^{2}+g_{1} \sin \phi\right. \\
\left.+g_{2} \cos (2 \phi)\right\}
\end{array}
$$

$g_{1} \sim \delta$ and $g_{2} \sim \alpha-\alpha_{c}$. The proportionality constants are of the order of the unity depending on the short range cutoff in the bosonization procedure. $\beta=\sqrt{2 \pi}$ for the isotropic model and $u$ is the spin wave velocity. The spectrum of this double frequency sine-Gordon (DFSG) field theory is not known in general. We start by analyzing both limits of $g_{1}=0$ and $g_{2}=0$ where the theory reduces to a single Sine-Gordon and a lot of information is disponible. In particular the semiclassical method of Dashen, Hasslasher and Neveu 12 gives the exact mass spectrum of the particles for this model.

For $g_{2}=0$ (we are indeed analyzing the whole zone $g_{2}<0$ because this marginal interaction renormalize to zero in this case) the excitation spectra consists of a kink, an antikink and two breathers. In the original spin language, the kink excitation carries $S_{z}=1$, the antikink $S_{z}=-1$ and the breathers $S_{z}=0$. The lower energy breather is degenerate with the kink and antikink and these three excitations give rise to a triplet branch, the correlate of the previously discussed magnon band. For $g_{1}=0$ the only one particle excitations of the resulting SG theory are a kink and an antikink, no breather is found. This kink carries spin one-half and is the analog of the massive spinons of the SS variational wave function. However note that in this parameter regime where the continuum approximation is reliable the characteristic width of these excitations is large compared with the lattice space.

We are now in position to discuss the excitations in the intermediate zone. We start by implementing a semiclassical calculation of the spectra of the theory (2). Even though the method is not expected to give exact results for this non integrable model, it gives a valuable non perturbative information which will allow us to interpolate between the desired limits. The time-independent equa- tion of motion corresponding to (2) is:

$$
\frac{u}{2 \beta^{2}}\left(\partial_{x}^{2} \phi_{0}\right)+g_{1} \cos \phi_{0}-2 g_{2} \sin 2 \phi_{0}=0
$$

The lowest energy (homogeneous) configuration is $\phi_{0}=$ $-\frac{\pi}{2}(\bmod 2 \pi)$ (it is not degenerate with $\phi_{0}=\frac{\pi}{2}$ as it would if $\left.g_{1}=0\right)$. Solitons are x-dependent solutions of the equation of motion with finite energy with respect to this homogeneous solution. Owing to the fact that the system is Lorentz invariant, moving solitons are obtained from the static ones by a Lorentz transformation. To look for these static configurations we write a first integral of Eq. (3):

$$
\frac{(1+B) \xi^{2}}{2}\left(\partial_{x} \phi_{0}\right)^{2}-B \sin \phi_{0}-\frac{1}{4} \cos 2 \phi_{0}=c t e
$$

where we have introducted the quantity $B=g_{1} /\left(4 g_{2}\right)$ as a characteristic parameter to analize the evolution of the spectrum from one limit to another. $\xi=u / \Delta$ and $\Delta=$ $\beta \sqrt{u\left(g_{1}+4 g_{2}\right)}$ is the mass gap to create a particle (let it call a meson) above the homogeneous vacuum. $\xi$ is the correlation length of the theory and measure the width of the kinks. The boundary conditions for finite energy are $\lim _{x \rightarrow \pm \infty} \phi_{0}=-\frac{\pi}{2}(\bmod 2 \pi)$ and $\lim _{x \rightarrow \pm \infty} \partial_{x} \phi_{0}=0$. They fix the cte $=B+\frac{1}{4}$. Equation (1) could now be integrated. The solution center at the origin is:

$$
\begin{aligned}
& t(x) \equiv \sin \left(\phi_{0}\right)=1-2 \cosh ^{2}\left[x_{0} / \xi\right] \operatorname{sech}\left[\left(x-x_{0}\right) / \xi\right] \\
& \operatorname{sech}\left[\left(x+x_{0}\right) / \xi\right]
\end{aligned}
$$

with

$$
x_{0}=\frac{1}{2} \log \left[\frac{2+B+\sqrt{1+B}}{B}\right]
$$

The quantity $x_{0}$ define a length scale which can be identified as the 'radius' of this excitations. In the limit of $B<<1$ we have $x_{0}>>1$ and $t(x)$ could be approximated by $\tanh \left(x-x_{0}\right) \tanh \left(x+x_{0}\right)$, i.e. a kink-antikink pair of the system without explicit dimerization. In the opposite limit $x_{0}=0$ and (5) becomes a kink solution of the static sine-Gordon equation corresponding to the dimerized chain. So that, $t(x)$ inter-poles between this limiting cases.

$\phi_{0}(x)=\arcsin [\mathrm{t}(\mathrm{x})]$ winds a complete round clockwise or counter-clockwise. Therefore this excitation carries $S_{z}= \pm 1$. The $S_{z}=0$ component of this triplet excitation could be taken into account in our semiclassical approach by including the periodic time-dependent solution of the field equation which cannot be obtained by a boosting of the static solution $t(x)$. After quantization, this solution will give additional singlet states which are not taken into account in the quantum states generated by the quantization of $t(x)$. For the single Sine Gordon equation these are the breather-like solutions. Furthermore, DHN have shown that the first breather is nothing but a renormalized meson. In this context the n-excited 
breather state is considered as bound state of $\mathrm{n}$ mesons. For our DFSG equation there are not analytic expressions for these breather solutions. We will consider in the following that a renormalized meson correspond to the $S_{z}=0$ component of our triplet excitation. That is to say, for the $\mathrm{SU}(2)$ invariant model we should expect that after an appropriated resumation of the perturbative series the meson mass $\Delta$ will be equal to the soliton mass to be introduced below.

To discuss the physical content of solution 5 we show in Fig. (1) the unstagered part of the local magnetization $<S_{i}^{z}>\left(\frac{1}{2 \pi} \partial_{x} \phi\right.$ in the bosonic representation) for different values of the ratio of the parameters B corresponding to $g_{1}$ smaller, equal or greater than $g_{2}$. The correlation length $(\xi)$ has been fixed to be ten times the lattice constant in this Figure. The figure have been obtained by freezing $\mathrm{t}(\mathrm{x})$. This corresponds to the physical situation where two nonmagnetic impurities cut the chain and fix the dimerization phase at the border. This situation have been recently studied by different numerical technicst. Note the similarities of the behavior showed in Fig. 1 and the one of Fig. 3a of ref. 11. For the clean translational invariant system the local magnetization is $\mathrm{x}$-independent as the result of the combination of this pattern center at the different site of the chain.

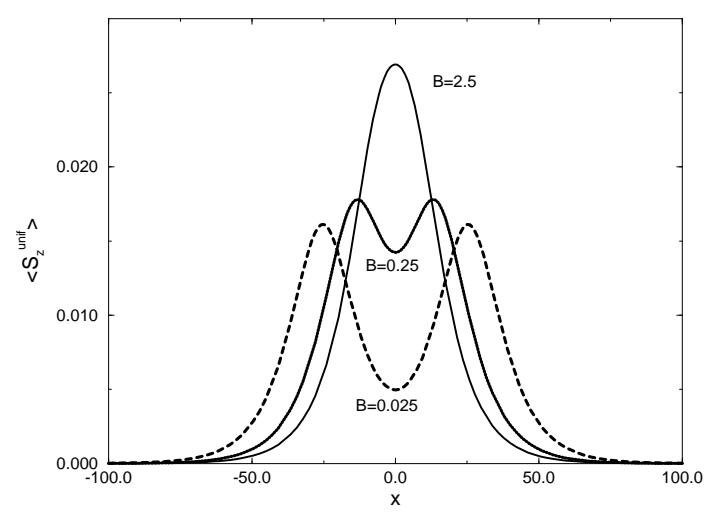

FIG. 1. Uniform part of the local magnetization carries by the configuration defined by $\mathrm{t}(\mathrm{x})$ for three values of the parameter B

Only for very low B there are two well separated peaks corresponding to two spin- $1 / 2$ excitations at a distance of the order $x_{0} / \xi$. For increasing B this two peaks are washed up and a single spin one excitation emerges at the chain center. All the intermediate spins in this configuration are excited from their value in the ground state. The excitation we are dealing up is therefore an extended spin-1 magnon which dissociate in two independent spinons for very weak dimerization and collapse in the magnon of the dimerized chain in the opposite strong dimerization limit. Note that at this leading order of our semiclassical expansion this is a rigid configuration which translates as a whole.

We now include gaussian fluctuation around this classical solution. The eigenvalues problem of the fluctuation operator corresponds to a Schroedinger-like equation which reads as:

$$
\begin{array}{r}
{\left[-\partial_{x}^{2}+V(x)\right] \psi=E \psi} \\
V(x)=-\frac{1}{1+B}\left[B t+\left(1-2 t(x)^{2}\right)\right]
\end{array}
$$

where we have made the substitution $x \rightarrow \frac{x}{\xi}$. The eigenfrequencies are given by $\omega=\Delta \sqrt{E}$. Equation (7) has a zero energy solution (with eigenfunction $\psi=\partial_{x} \phi_{0}$ ), this is connected with the translational mode arising in the broken of translational invariance of $t(x)$. We recall that this is the only bound state of the fluctuation operator for the case of the single sine-Gordon solitons. For the present case we find in addition another bound state which split from this zero mode for finite $B$. For increasing $B$ the energy of this mode increases going to the continuum spectra of $V(x)$ for $B \rightarrow \infty$. In Fig. 2 we show the evolution of this eigenvalue with $B$. This figure has been obtained by numerical integration of Eq. (7).

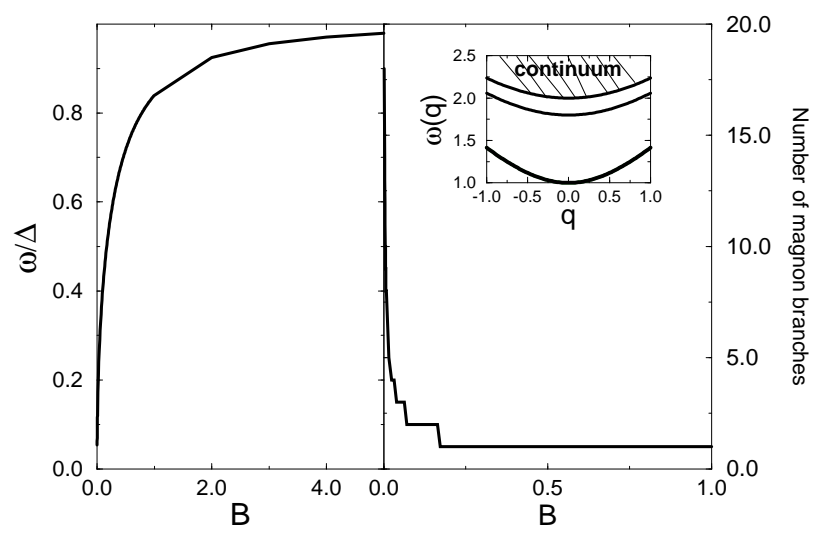

FIG. 2. (a) The evolution of the non-zero eigenfrequency of the fluctuation operator as obtained by numerical integration of (7). (b) The number of magnon branches predicted by the semiclassical calculation. The insertion shows a typical low energy spectrum.

The excitation spectra of the theory in the sector of $S z= \pm 1$ is spanned by the following states:

- The state of the quantum particle (of mass M) builded around $t(x)$. We identify this as belonging to a one-magnon branch with dispersion $E(p)=$ $u \sqrt{p^{2}+M^{2}}$

- The excited states of the magnon of mass $M^{*}=$ $M+n \omega$. There are as much as adittional triplet 
branches as $n \omega$ reaches the value $\Delta$ where the continuum of the Schroedinger equation starts. This continuum corresponds to:

- Labeling by q the continuum of level of Eq. (7), they are $\omega(q)=u \sqrt{q^{2}+\frac{1}{\xi^{2}}}$ This is just the kinetic energy of a meson with momentum q. Therefore this state corresponds to the scattering of a meson in presence of the soliton. Moreover, when one of the continuum modes is excited once, we get a two particle meson-soliton state. As we have associated a meson with the elementary $S_{z}=0$ excitation of the theory these excitations correspond to the $S_{z}=1$ component of the two magnon continuum of our original spin chain.

The semiclassical calculation predicts the apparition of additional equi-spaciated magnon branches. The number of these additional branches diverges as $\mathrm{B}$ goes to zero; this is nothing but the two spinons continuum of the undimerized chain. For decreasing dimerization or increasing frustration the number of the excited magnon states decrease until value of $B$. Beyond this critical value only two magnon branches are found. In Fig. 2 (b) we show the behavior previously discussed. The insertion gives our prediction for the low energy spectra for $\mathrm{B}$ greater than the critical value. Previous exact diagonalization studies of the spectra of Hamiltonian 1 hafe shown the apparition of an additional triplet branch $\mathbf{7}, 2$ confirming this picture. The increase of the number of the triplet branches with $\delta$ has also recently seen in numerical studies of this system $\mathbb{t}$.

Now, we address the question of the contact of this semiclassical approach and previous work assuming a linear confinement potential between the spinons.

For $B<<1$ (weak dimerization limit) it is customary to think the problem as the onp of two interacting kinks as has been recently proposed 3 .1. If they are well separated their interaction is given by an attractive linear potential, the one arising on the term proportional to $g_{1}$ in Eq. (2). At low energy the spectra of the system corresponds to the one of two particle interacting via this linear potential. A ladder of bound states is obtained by solution of an effective Schroedinger equation. At high enough energy these bound states are truncated because it becomes favorable to create a new kink-antikink pair than to excite one of them. This is essentially the scenario discussed in ref. (3)

Let us define an effective kink-antikink potential by the following procedure: take Eq.(5) as a suitable kinkantikink form with $x_{0}$ a variable distance, replace this form in Eq. (2) so the $x_{0}$-dependence of the total energy gives our kink-antikink interaction energy. The result is show in Fig. (3) for two small values of B where the kinks are expected to be well defined excitations.

Two competing interactions could be clearly identify. An attractive linear interaction and a repulsive one acting at small distance. The repulsive interaction is originated on the finite width of the kinks. The value of $x_{0}$ given in eq. (6) represent the minimuun of the total potential. For very small B the slope of the linear potential is small and this minimum is not well defined. The kinks and antikinks will be always far apart and they never seen the barrier. Therefore the linear approximation for the kink-antikink potential will give essentially the same result as a potential like the one showed in the figure. The semiclassical approach gives only poor quantitative results in this zone because it describes the problem as the one of an harmonic oscillators with very small frequency. However the general qualitative behavior of the spectra is well reproduced, i.e. the divergence of the number of excited states in the limit of no dimerization.

For increasing B, a well defined minimum appears in the potential energy. Now, at low energy the kinkantikink oscillates around this minimum as bounded by a spring. Our system behaves as a vibrating molecule made by a kink and an antikink. The semiclassical approximation previously discussed precisely describes the quantum state of this harmonic oscillator.

By further increasing B the kinks loose their identity, $\mathrm{t}(\mathrm{x})$ becomes almost a kink solution of the SG theory with $g_{2}=0$. The semiclassical result represents an internal oscilation mode in which the kink waveform undergoes a harmonically varying shape change localized about the kink center.

Note that even for very small B the kinks are not well separated in the order of their width, thererofe we should associate the excitation in a broad range of the parameter as a unique magnon dispersed over a great number of spins.

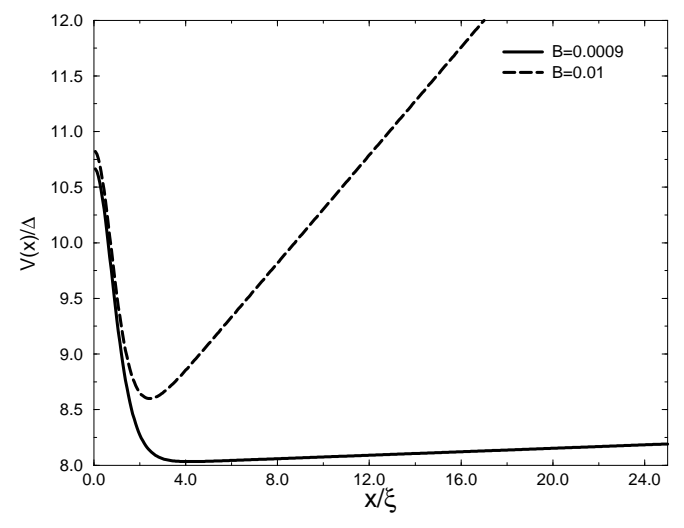

FIG. 3. kink-antikink interaction potential as defined in the text

Finally we analyze the experimental situation in $\mathrm{CuGeO}_{3}$. Neutron scattering measurements of the magnetic spectra 13 shows only one dispersive excitation before the continuum. The excited states previously discussed has not been found in these experiments. Two 
possible reasons could be given. As it has recently show by numerical diagonalization the spectral weight of these states are very small. Moreover, in the original spin-phonon-model the inclusion of the interchain elastic coupling 10 implies that spins interact with transversal acoustic phonons. Therefore this excited state could decay in acoustic phonons so broadening the magnon peak. More experimental and theoretical work will be needed to elucidate this point.

We thank I. Affleck for useful discussions and J. Riera for critical reading of the manuscript. A. D. acknowledges Fundación Antorchas for financial support.

${ }^{1}$ E. Sorensen, I. Affleck, D. Augier and D. Poiblanck , preprint condmat/9804085. The interaction spinonimpurity have been analized in D. Augier, P. Hansen, D. Poilblanc, J. Riera, and E. Sorensen, cond-mat/9807265 and G. Uhrig, F. Schonfeld, M. Laukamp and E. Dagotto condmat/9805245.

${ }^{2}$ G. Bouzerar, A.P. Kampf and G.I. Japaridze, preprint condmat/9801046

${ }^{3}$ I. Affleck, in Dynamical Properties of Unconventional Magnetic Systems (NATO ASI, Geilo, Norway, 1997) and condmat/9705127.

${ }^{4}$ M. Hase, I. Terasaki and K. Uchinokura, Phys. Rev. Lett. 70, 3651 (1993).

${ }^{5}$ J. Riera and A. Dobry, Phys. Rev. B 51, 16098 (1995).

${ }^{6}$ G. Castilla, S. Chakravarty and V.J. Emery, Phys. Rev. Lett. 75, 1823 (1995).

${ }^{7}$ J. Riera and S. Koval, Phys. Rev. B 53, 770 (1996)

${ }^{8}$ G. S. Uhrig and H. J. Schulz, Phys. Rev. B 54, R9624 (1996).

${ }^{9}$ D. Augier, D. Poiblanc, E. Sorensen and I. Affleck, condmat/9802053

${ }^{10}$ A. Dobry and D. Ibaceta, Phys. Rev. B 58, 3124 (1998)

${ }^{11}$ B.S. Shatry and B. Sutterland, Phys. Rev. Lett. 47, 964 (1981)

12 R. Dashen, B. Hasslacher and A. Neveu, Phys. Rev. D 11 , 3424 (1975)

${ }^{13}$ M. Ä̈n et al., Phys. Rev. Lett. 78, 1560 (1997).

${ }^{14}$ I. Affleck, Fields Strings and Critical Phenomena, edited by E. Brézin and J.Zinn-Justin (North-Holland, Amsterdam 1990), pg. 563. 